\title{
Evolutionary models of rotating dense stellar systems: challenges in software and hardware
}

\author{
Jose Fiestas \\ National Astronomical Observatories of China, Chinese Academy of Sciences, Datun Lu 20A, \\ Chaoyang District, Beijing 100012, China \\ email: fiestas@nao.cas.cn
}

\begin{abstract}
We present evolutionary models of rotating self-gravitating systems (e.g. globular clusters, galaxy cores). These models are characterized by the presence of initial axisymmetry due to rotation. Central black hole seeds are alternatively included in our models, and black hole growth due to consumption of stellar matter is simulated until the central potential dominates the kinematics in the core. Goal is to study the long-term evolution ( $\sim$ Gyr) of relaxed dense stellar systems, which deviate from spherical symmetry, their morphology and final kinematics. With this purpose, we developed a 2D Fokker-Planck analytical code, which results we confirm by detailed N-Body techniques, applying a high performance code, developed for GPU machines. We compare our models to available observations of galactic rotating globular clusters, and conclude that initial rotation modifies significantly the shape and lifetime of these systems, and can not be neglected in studying the evolution of globular clusters, and the galaxy itself.
\end{abstract}

Keywords. methods: numerical - gravitation - stellar dynamics - black hole - globular clusters: general - galactic nuclei

\section{Systems without black hole}

As initial models, we use rotating King Models. We select a set of galactic Globular Clusters which are flattened by rotation, and apply the technique of Fiestas et.al. (2006) in order to find initial configurations for these systems. Figure 1 shows simulation data (symbols without error bars), observational data (symbols with error bars), together with curves of isotropic rotating spheroids (solid lines).

Observations show in many cases clear deviations from isotropic rotators from which our models do not deviate significantly. Note that final configurations are a consequence of dynamical relaxation, and angular momentum transfer. It shows that only in a few cases, ellipticity is determined by rotation, while in other cases anisotropy and/or tidal effects modify the cluster shape. More studies are being done in order to rule out observation uncertainties, which will be target of our future simulations.

\section{Systems with black hole}

The gravitational dominance of the central BH of mass $M_{\mathrm{bh}}$ over the surrounding stars with velocity dispersion $\sigma_{\mathrm{c}}$ vanishes at a radius defined by

$$
r_{\mathrm{h}}=\frac{G M_{\mathrm{bh}}}{\sigma_{\mathrm{c}}^{2}}
$$

Our simulations show that rotation grows inside $r_{\mathrm{h}}$, what can be explain through capture of strong rotating massive stars by the $\mathrm{BH}$, and angular momentum transfer happening from the lighter to the massive component as a consequence of dynamical relaxation. 


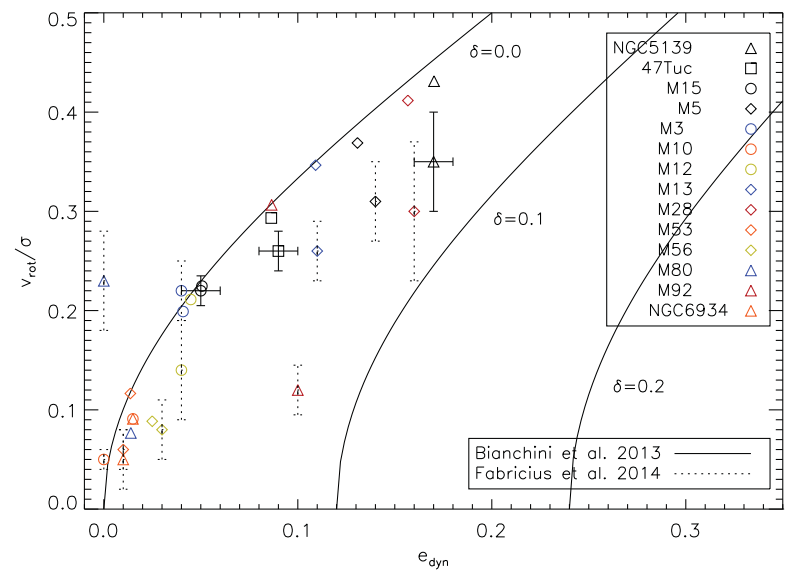

Figure 1. $v_{\text {rot }} / \sigma$ against ellipticity for selected galactic globular clusters

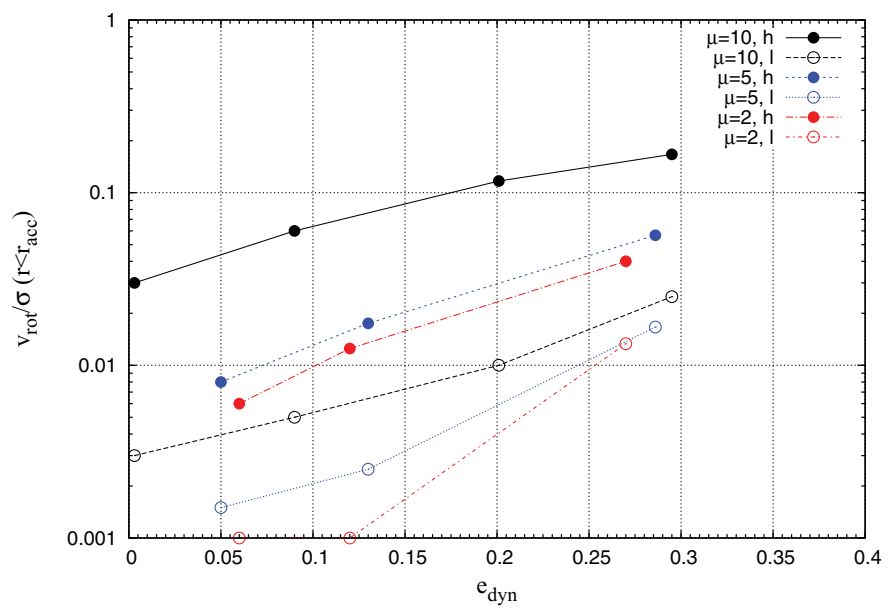

Figure 2. $v_{\text {rot }} / \sigma$ for all $\mathrm{BH}$ models

Figure 2 shows final rotation of simulation data inside rh vs. dynamical ellipticity for different individual mass rates $\mu=m_{h} / m_{l}$ for heavy (filled circles) and light (open circles) stars. Symbols are connected with lines, along which initial rotation increases from left to right. This correlation is a consequence of angular momentum transfer, enhanced by relaxation processes, which maintain $v_{\text {rot }}$ increasing towards the center. Although the obtained $v_{\text {rot }} / \sigma$ in our set of models is difficult to detect by current observational techniques, ellipticity in galactic cores or globular star clusters is a measurable quantity, which can be used to detect this feature, and give conclusions about the formation and evolution of systems harboring central BHs.

\section{References}

Bahcall J. N. \& Wolf R. A. 1976, ApJ, 209, 214

Fiestas J., Spurzem R. \& Kim E. 2006, MNRAS, 373, 677

Fiestas J. \& Spurzem R. 2010, MNRAS, 405, 194

Fiestas J., Porth O. \& Spurzem R. 2012, MNRAS 419, 57 